Supplement of Biogeosciences Discuss., 12, 12691-12712, 2015

http://www.biogeosciences-discuss.net/12/12691/2015/

doi:10.5194/bgd-12-12691-2015-supplement

(C) Author(s) 2015. CC Attribution 3.0 License.

(c) (i)

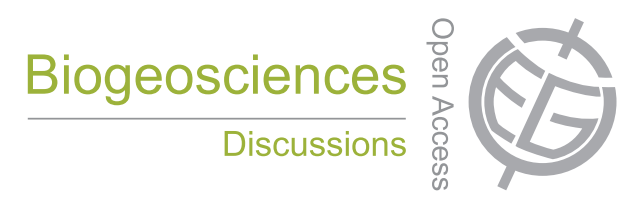

Supplement of

\title{
Phytoplankton calcification as an effective mechanism to prevent cellular calcium poisoning
}

M. N. Müller et al.

Correspondence to: M. N. Müller (mnmuller@usp.br)

The copyright of individual parts of the supplement might differ from the CC-BY 3.0 licence. 
Table S1: Seawater chemistry of the first experiment. TA = Total Alkalinity. DIC $=$ Dissolved Inorganic Carbon. Omega = Calcium carbonate saturation state.

\begin{tabular}{|c|c|c|c|c|c|c|}
\hline Species & $\mathrm{Ca}(\mathrm{mmol} / \mathrm{L})$ & $\mathrm{TA}(\mu \mathrm{mol} / \mathrm{kg})$ & $\mathrm{DIC}(\mu \mathrm{mol} / \mathrm{kg})$ & $\mathrm{pH}$ (total scale) & 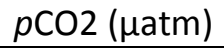 & Omega (calcite) \\
\hline Chaetoceros sp. & 1 & $2387 \pm 9$ & $2042 \pm 1$ & $8.18 \pm 0.01$ & $300 \pm 7$ & $1.0 \pm 0.1$ \\
\hline Chaetoceros sp. & 9 & $2376 \pm 4$ & $2050 \pm 45$ & $8.15 \pm 0.07$ & $322 \pm 49$ & $4.7 \pm 2.2$ \\
\hline Chaetoceros sp. & 19 & $2404 \pm 1$ & $2074 \pm 20$ & $8.16 \pm 0.03$ & $319 \pm 20$ & $6.4 \pm 1.0$ \\
\hline Chaetoceros sp. & 26 & $2391 \pm 3$ & $2074 \pm 27$ & $8.13 \pm 0.05$ & $343 \pm 32$ & $6.8 \pm 1.3$ \\
\hline Chaetoceros sp. & 34 & $2377 \pm 3$ & $2061 \pm 8$ & $8.12 \pm 0.02$ & $385 \pm 8$ & $7.2 \pm 0.4$ \\
\hline Chaetoceros sp. & 42 & $2397 \pm 7$ & $2061 \pm 12$ & $8.16 \pm 0.01$ & $318 \pm 6$ & $8.3 \pm 0.6$ \\
\hline Species & $\mathrm{Ca}(\mathrm{mmol} / \mathrm{L})$ & $\mathrm{TA}(\mu \mathrm{mol} / \mathrm{kg})$ & $\mathrm{DIC}(\mu \mathrm{mol} / \mathrm{kg})$ & $\mathrm{pH}$ (total scale) & 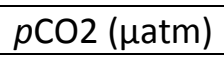 & Omega (calcite) \\
\hline H. akashiwo & 1 & $2379 \pm 1$ & $2083 \pm 34$ & $8.10 \pm 0.06$ & $365 \pm 43$ & $0.9 \pm 1.6$ \\
\hline H. akashiwo & 9 & $2345 \pm 5$ & $2021 \pm 21$ & $8.14 \pm 0.04$ & $355 \pm 19$ & $4.5 \pm 1.0$ \\
\hline H. akashiwo & 19 & $2415 \pm 2$ & $2116 \pm 9$ & $8.09 \pm 0.02$ & $409 \pm 11$ & $5.6 \pm 0.5$ \\
\hline H. akashiwo & 26 & $2427 \pm 0$ & $2113 \pm 11$ & $8.13 \pm 0.02$ & $343 \pm 14$ & $6.9 \pm 0.5$ \\
\hline H. akashiwo & 34 & $2369 \pm 1$ & $2129 \pm 4$ & $7.99 \pm 0.01$ & $481 \pm 10$ & $5.6 \pm 0.2$ \\
\hline H. akashiwo & 42 & $2377 \pm 2$ & $2156 \pm 4$ & $7.94 \pm 0.04$ & $576 \pm 34$ & $5.5 \pm 0.2$ \\
\hline Species & $\mathrm{Ca}(\mathrm{mmol} / \mathrm{L})$ & $\mathrm{TA}(\mu \mathrm{mol} / \mathrm{kg})$ & $\mathrm{DIC}(\mu \mathrm{mol} / \mathrm{kg})$ & $\mathrm{pH}$ (total scale) & 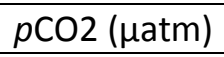 & Omega (calcite) \\
\hline Cylindrotheca sp. & 10 & $2061 \pm 5$ & $1724 \pm 8$ & $8.13 \pm 0.02$ & $279 \pm 14$ & $3.8 \pm 0.5$ \\
\hline Cylindrotheca sp. & 22 & $2089 \pm 11$ & $1742 \pm 7$ & $8.14 \pm 0$ & $272 \pm 2$ & $5.1 \pm 0.4$ \\
\hline Cylindrotheca sp. & 34 & $2098 \pm 10$ & $1806 \pm 7$ & $8.04 \pm 0.01$ & $363 \pm 12$ & $4.9 \pm 0.4$ \\
\hline Cylindrotheca sp. & 45 & $2131 \pm 3$ & $1853 \pm 5$ & $8.01 \pm 0.01$ & $406 \pm 14$ & $5.1 \pm 0.3$ \\
\hline Cylindrotheca sp. & 52 & $2133 \pm 0$ & $1816 \pm 0$ & $8.09 \pm 0$ & $324 \pm 0$ & $6.4 \pm 0$ \\
\hline Species & $\mathrm{Ca}(\mathrm{mmol} / \mathrm{L})$ & $\mathrm{TA}(\mu \mathrm{mol} / \mathrm{kg})$ & $\mathrm{DIC}(\mu \mathrm{mol} / \mathrm{kg})$ & $\mathrm{pH}$ (total scale) & 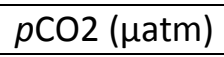 & Omega (calcite) \\
\hline E. huxleyi (naked) & 1 & $2490 \pm 3$ & $2112 \pm 2$ & $8.23 \pm 0.01$ & $274 \pm 4$ & $1.1 \pm 0.1$ \\
\hline E. huxleyi (naked) & 9 & $2497 \pm 6$ & $2100 \pm 8$ & $8.25 \pm 0.01$ & $256 \pm 3$ & $5.9 \pm 0.4$ \\
\hline E. huxleyi (naked) & 19 & $2516 \pm 7$ & $2165 \pm 5$ & $8.19 \pm 0.02$ & $300 \pm 11$ & $7.1 \pm 0.3$ \\
\hline E. huxleyi (naked) & 26 & $2492 \pm 5$ & $2062 \pm 4$ & $8.30 \pm 0$ & $227 \pm 2$ & $9.6 \pm 0.2$ \\
\hline
\end{tabular}




\begin{tabular}{|c|c|c|c|c|c|c|}
\hline E. huxleyi (naked) & 34 & $2520 \pm 3$ & $2133 \pm 8$ & $8.23 \pm 0.01$ & $286 \pm 6$ & $9.3 \pm 0.4$ \\
\hline E. huxleyi (naked) & 42 & $2533 \pm 5$ & $2100 \pm 4$ & $8.30 \pm 0.01$ & $227 \pm 3$ & $11.2 \pm 0.2$ \\
\hline Species & $\mathrm{Ca}(\mathrm{mmol} / \mathrm{L})$ & $\mathrm{TA}(\mu \mathrm{mol} / \mathrm{kg})$ & $\mathrm{DIC}(\mu \mathrm{mol} / \mathrm{kg})$ & $\mathrm{pH}$ (total scale) & 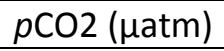 & Omega (calcite) \\
\hline E. huxleyi (calcified) & 1 & $2359 \pm 2$ & $2114 \pm 13$ & $8.01 \pm 0.02$ & $454 \pm 24$ & $0.7 \pm 0.6$ \\
\hline E. huxleyi (calcified) & 9 & $2322 \pm 2$ & $2051 \pm 2$ & $8.07 \pm 0$ & $386 \pm 4$ & $3.9 \pm 0.1$ \\
\hline E. huxleyi (calcified) & 21 & $2412 \pm 4$ & $2141 \pm 2$ & $8.06 \pm 0$ & $415 \pm 2$ & $5.3 \pm 0.1$ \\
\hline E. huxleyi (calcified) & 25 & $2421 \pm 8$ & $2066 \pm 15$ & $8.21 \pm 0.03$ & $272 \pm 22$ & $8.0 \pm 0.7$ \\
\hline E. huxleyi (calcified) & 40 & $2351 \pm 3$ & $2105 \pm 5$ & $8.02 \pm 0.02$ & $447 \pm 15$ & $5.9 \pm 0.2$ \\
\hline E. huxleyi (calcified) & 52 & $2350 \pm 10$ & $2089 \pm 13$ & $8.05 \pm 0.04$ & $416 \pm 35$ & $6.7 \pm 0.6$ \\
\hline Species & $\mathrm{Ca}(\mathrm{mmol} / \mathrm{L})$ & $\mathrm{TA}(\mu \mathrm{mol} / \mathrm{kg})$ & $\mathrm{DIC}(\mu \mathrm{mol} / \mathrm{kg})$ & pH (total scale) & 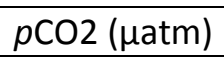 & Omega (calcite) \\
\hline G. oceanica & 1 & $2479 \pm 3$ & $2126 \pm 3$ & $8.19 \pm 0$ & $291 \pm 3$ & $1.0 \pm 0.2$ \\
\hline G. oceanica & 9 & $2445 \pm 29$ & $2109 \pm 5$ & $8.17 \pm 0.05$ & $304 \pm 37$ & $5.0 \pm 0.3$ \\
\hline G. oceanica & 21 & $2444 \pm 6$ & $2096 \pm 10$ & $8.19 \pm 0.01$ & $288 \pm 10$ & $6.8 \pm 0.5$ \\
\hline G. oceanica & 25 & $2444 \pm 2$ & $2081 \pm 9$ & $8.22 \pm 0.01$ & $270 \pm 9$ & $8.2 \pm 0.4$ \\
\hline G. oceanica & 40 & $2452 \pm 2$ & $2140 \pm 13$ & $8.13 \pm 0.02$ & $345 \pm 15$ & $7.6 \pm 0.6$ \\
\hline G. oceanica & 52 & $2467 \pm 10$ & $2091 \pm 3$ & $8.23 \pm 0.01$ & $259 \pm 5$ & $9.7 \pm 0.2$ \\
\hline
\end{tabular}

